\title{
Some Properties of Furuta Type Inequalities and Applications
}

\author{
Jiangtao Yuan and Caihong Wang \\ School of Mathematics and Information Science, Henan Polytechnic University, Jiaozuo, Henan 454000, China \\ Correspondence should be addressed to Jiangtao Yuan; jtyuan@hpu.edu.cn
}

Received 25 January 2014; Accepted 20 March 2014; Published 13 April 2014

Academic Editor: Changsen Yang

Copyright (C) 2014 J. Yuan and C. Wang. This is an open access article distributed under the Creative Commons Attribution License, which permits unrestricted use, distribution, and reproduction in any medium, provided the original work is properly cited.

\begin{abstract}
This work is to consider Furuta type inequalities and their applications. Firstly, some Furuta type inequalities under $A \geq B \geq 0$ are obtained via Loewner-Heinz inequality; as an application, a proof of Furuta inequality is given without using the invertibility of operators. Secondly, we show a unified satellite theorem of grand Furuta inequality which is an extension of the results by Fujii et al. At the end, a kind of Riccati type operator equation is discussed via Furuta type inequalities.
\end{abstract}

\section{Introduction}

Throughout this paper, an operator $T$ means a bounded linear operator on a Hilbert space. $T \geq 0$ and $T>0$ mean a positive operator and an invertible positive operator, respectively, (see [1, page 103]). The classical Loewner-Heinz inequality (L-H) is stated below (see [2, page 127]).

Theorem 1 (Loewner-Heinz inequality (L-H)). Let $p \in[0,1]$; then $A \geq B \geq 0$ ensures

$$
A^{p} \geq B^{p} .
$$

In general, $(\mathrm{L}-\mathrm{H})$ is not true for $p>1$. As a celebrated development of (L-H), Furuta provided a kind of order preserving operator inequality [2, page 129], the so-called Furuta inequality (FI).

Theorem 2 (Furuta inequality (FI), [3]). Let $r \geq 0, p>0$; then $A \geq B \geq 0$ ensures

$$
\begin{aligned}
& \left(B^{r / 2} A^{p} B^{r / 2}\right)^{(\min \{1, p\}+r) /(p+r)} \geq\left(B^{r / 2} B^{p} B^{r / 2}\right)^{(\min \{1, p\}+r) /(p+r)}, \\
& \left(A^{r / 2} A^{p} A^{r / 2}\right)^{(\min \{1, p\}+r) /(p+r)} \geq\left(A^{r / 2} B^{p} A^{r / 2}\right)^{(\min \{1, p\}+r) /(p+r)} .
\end{aligned}
$$

Tanahashi proved that the outer exponent $\min \{1, p\}+r$ above is optimal; see [3] for related topics. In order to establish the order structure on Aluthge transform of nonnormal operators, the complete form of Furuta inequality was showed in [4].
Theorem 3 (Complete form [4]). Let $q>0, r \geq 0, p>p_{0}>0$ and $s(q)=\min \left\{p, 2 p_{0}+\min \{q, r\}\right\}$. Then $A \geq 0$ and $B \geq 0$ such that $A^{q} \geq B^{q}$ ensures

$$
\left(A^{r / 2} B^{p_{0}} A^{r / 2}\right)^{(s(q)+r) /\left(p_{0}+r\right)} \geq\left(A^{r / 2} B^{p} A^{r / 2}\right)^{(s(q)+r) /(p+r)} .
$$

We call the theorem above the complete form of Furuta inequality because the case $p_{0}=q=1$ of it implies the essential part $(p>1)$ of Furuta inequality by the LoewnerHeinz inequality for $(1+r) /(s(1)+r) \in(0,1]$. For convenience, we call Furuta inequality (Theorem 2 ) the original form of Furuta inequality.

It is known that there are many applications of Furuta type inequalities; we cite [5-7].

Based on Ito et al. [8] which is a continuation of [9], the equivalent relations between two operator inequalities are useful. For $A \geq 0, A^{0}$ means the projection $P_{(\operatorname{ker} A)^{\perp}}$.

Theorem 4 (see [8]). Let $r>0,0 \leq p_{0}<p, A \geq 0$ and $B \geq 0$.

(1) If $\operatorname{ker}\left(A B^{p_{0} / 2}\right) \subseteq \operatorname{ker} B$, then, for each $r, p_{0}$, and $p$, the following inequalities are equivalent to each other:

$$
\begin{aligned}
& \left(B^{p / 2} A^{r} B^{p / 2}\right)^{\left(p-p_{0}\right) /(r+p)} \geq\left(B^{p / 2} B^{r} B^{p / 2}\right)^{\left(p-p_{0}\right) /(r+p)}, \\
& \left(A^{r / 2} B^{p_{0}} A^{r / 2}\right)^{\left(p_{0}+r\right) /\left(p_{0}+r\right)} \geq\left(A^{r / 2} B^{p} A^{r / 2}\right)^{\left(p_{0}+r\right) /(p+r)} .
\end{aligned}
$$

In particular, (4) implies (5) without condition $\operatorname{ker}\left(A B^{p_{0} / 2}\right) \subseteq \operatorname{ker} B$. 
(2) For each $r, p_{0}$, and $p$, the following inequalities are equivalent to each other:

$$
\begin{aligned}
& \left(A^{p / 2} B^{r} A^{p / 2}\right)^{\left(p-p_{0}\right) /(r+p)} \leq\left(A^{p / 2} A^{r} A^{p / 2}\right)^{\left(p-p_{0}\right) /(r+p)}, \\
& \left(B^{r / 2} A^{p_{0}} B^{r / 2}\right)^{\left(p_{0}+r\right) /\left(p_{0}+r\right)} \leq\left(B^{r / 2} A^{p} B^{r / 2}\right)^{\left(p_{0}+r\right) /(p+r)} .
\end{aligned}
$$

It should be pointed out that (5) ensures (4) is not true without the condition $\operatorname{ker}\left(A B^{p_{0} / 2}\right) \subseteq \operatorname{ker} B$ [8, Remark 1]. Moreover, the proof of Theorem 4 is independent of $(\mathrm{L}-\mathrm{H})$.

In Section 2, some Furuta type inequalities under $A \geq$ $B \geq 0$ are proved via Loewner-Heinz inequality; as applications, we show alternate proofs of some well-known Furuta type inequalities (proofs of Theorems 10 and 2).

In 1995, Furuta [10] proved the so-called grand Furuta inequality which is also an extension of Theorem 2.

Theorem 5 (grand Furuta inequality [10]). Let $p \geq 1,-1 \leq$ $t<0, r \geq-t$ and $s \geq 1$. If $A \geq B \geq 0$ with $A>0$; then

$$
A^{1+t+r} \geq\left(A^{r / 2}\left(A^{t / 2} B^{p} A^{t / 2}\right)^{s} A^{r / 2}\right)^{(1+t+r) /((p+t) s+r)} .
$$

Fujii et al. proved some satellite theorems of grand Furuta inequality.

Theorem 6 (see [11]). Let $p \geq 1,-1 \leq t<0, r \geq-t$ and $s \geq 1$. If $A \geq B \geq 0$ with $A>0$; then

$$
\left(A^{r / 2}\left(A^{t / 2} B^{p} A^{t / 2}\right)^{s} A^{r / 2}\right)^{(1+t+r) /((p+t) s+r)} \leq A^{(r+t) / 2} B A^{(r+t) / 2} .
$$

Theorem 7 (see [12]). Let $p \geq 1,-1 \leq t<0, r \geq-t$ and $s \geq 1$. If $A \geq B \geq 0$ with $A>0$; then

$$
\begin{gathered}
\left(A^{r / 2}\left(A^{t / 2} B^{p} A^{t / 2}\right)^{s} A^{r / 2}\right)^{(1+t+r) /((p+t) s+r)} \\
\leq A^{r / 2}\left(A^{t / 2} B^{p} A^{t / 2}\right)^{(1+t) /(p+t)} A^{r / 2} .
\end{gathered}
$$

Theorems 6 and 7 are extensions of Theorem 5 .

In Section 3, we will show a unified satellite theorem which is an extension of Theorems 6 and 7 via the complete forms of Furuta inequality with negative powers.

Lastly, it is known that Riccati type operator equations $K=T H T-T C-C^{*} T$ relate to control theory closely and have been studied extensively [13]. Pedersen and Takesaki [14] developed the special kind of Riccati equation $K=T H T$ as a useful tool for the noncommutative Radon-Nikodym theorem.

Yuan and Gao [15] discussed the Riccati type equation:

$$
K^{p}=H^{\delta / 2} T^{1 / 2}\left(T^{1 / 2} H^{\delta+r} T^{1 / 2}\right)^{(p-\delta) /(\delta+r)} T^{1 / 2} H^{\delta / 2} .
$$

In Section 4 , as a continuation of $[15,16]$, we will consider the Riccati type equation:

$$
K^{p}=H^{\delta / 2} T^{1 / 2}\left(T^{1 / 2} H^{\delta+r} T^{1 / 2}\right)^{(1 / w)-1} T^{1 / 2} H^{\delta / 2}
$$

via Furuta type inequalities.

\section{Furuta Type Inequalities under the Order $A \geq B \geq 0$}

Reference [17] proved a kind of equivalent relations which can be regarded as a parallel result to Theorem 4 .

Theorem 8 (see [17]). Let $r>0,0<p_{0}<p, A \geq 0$ and $B \geq 0$. If $\operatorname{ker}\left(A B^{p_{0} / 2}\right) \subseteq \operatorname{ker} B$, then, for each $r, p_{0}$ and $p$, the following inequalities are equivalent to each other:

$$
\begin{gathered}
\left(B^{p_{0} / 2} A^{r} B^{p_{0} / 2}\right)^{\left(p-p_{0}\right) /\left(r+p_{0}\right)} \\
\geq(\text { resp. } \leq)\left(B^{p_{0} / 2} B^{r} B^{p_{0} / 2}\right)^{\left(p-p_{0}\right) /\left(r+p_{0}\right)}, \\
\left(A^{r / 2} B^{p_{0}} A^{r / 2}\right)^{(p+r) /\left(p_{0}+r\right)} \\
\geq(\text { resp. } \leq)\left(A^{r / 2} B^{p} A^{r / 2}\right)^{(p+r) /(p+r)} .
\end{gathered}
$$

In particular, (12) implies (13) without condition $\operatorname{ker}\left(A B^{p_{0} / 2}\right) \subseteq$ ker $B$.

The proof of Theorem 8 is different from Theorem 4 and independent of $(\mathrm{L}-\mathrm{H})$.

In this section, we consider some Furuta type inequalities under the order $A \geq B \geq 0$. As applications, alternate proofs of some Furuta type inequalities are given (proofs of Theorems 10 and 2). Especially, we prove (FI) without using the invertibility of operators.

Theorem 9. Let $1 \geq r>0, A \geq B \geq 0$.

(1) For each $p_{0}>0$ and $p$ with $p_{0}<p \leq 2 p_{0}+r$, the following inequalities hold and they are equivalent to each other:

$$
\begin{aligned}
& \left(B^{p_{0} / 2} A^{r} B^{p_{0} / 2}\right)^{\left(p-p_{0}\right) /\left(r+p_{0}\right)} \geq\left(B^{p_{0} / 2} B^{r} B^{p_{0} / 2}\right)^{\left(p-p_{0}\right) /\left(r+p_{0}\right)}, \\
& \left(A^{r / 2} B^{p_{0}} A^{r / 2}\right)^{(p+r) /\left(p_{0}+r\right)} \geq\left(A^{r / 2} B^{p} A^{r / 2}\right)^{(p+r) /(p+r)} .
\end{aligned}
$$

(2) For each $p_{0}>0$ and $p$ with $p_{0}<p \leq 2 p_{0}+r$, the following inequalities hold:

$$
\begin{aligned}
\left(A^{p_{0} / 2} B^{r} A^{p_{0} / 2}\right)^{\left(p-p_{0}\right) /\left(r+p_{0}\right)} & \leq\left(A^{p_{0} / 2} A^{r} A^{p_{0} / 2}\right)^{\left(p-p_{0}\right) /\left(r+p_{0}\right)} \\
\left(B^{r / 2} A^{p_{0}} B^{r / 2}\right)^{(p+r) /\left(p_{0}+r\right)} & \leq\left(B^{r / 2} A^{p} B^{r / 2}\right)^{(p+r) /(p+r)}
\end{aligned}
$$

(3) If $p>1$, then

$$
\begin{aligned}
& A^{1+r} \geq A^{r / 2} B A^{r / 2} \geq\left(A^{r / 2} B^{p} A^{r / 2}\right)^{(1+r) /(p+r)}, \\
& B^{1+r} \leq B^{r / 2} A B^{r / 2} \leq\left(B^{r / 2} A^{p} B^{r / 2}\right)^{(1+r) /(p+r)} .
\end{aligned}
$$


Proof. (1) Since $p_{0}<p \leq 2 p_{0}+r, 1 \geq\left(p-p_{0}\right) /\left(r+p_{0}\right)>0$ follows. By $A \geq B \geq 0$ and (L-H) for $1 \geq r>0$ and $1 \geq$ $\left(p-p_{0}\right) /\left(r+p_{0}\right)>0$, we have

$$
\begin{gathered}
A^{r} \geq B^{r}, \quad B^{p_{0} / 2} A^{r} B^{p_{0} / 2} \geq B^{p_{0} / 2} B^{r} B^{p_{0} / 2}, \\
\left(B^{p_{0} / 2} A^{r} B^{p_{0} / 2}\right)^{\left(p-p_{0}\right) /\left(r+p_{0}\right)} \geq\left(B^{p_{0} / 2} B^{r} B^{p_{0} / 2}\right)^{\left(p-p_{0}\right) /\left(r+p_{0}\right)} .
\end{gathered}
$$

Hence, (14) holds. Since $A \geq B \geq 0, \operatorname{ker}\left(A B^{p_{0} / 2}\right) \subseteq \operatorname{ker} B$ follows. So, the equivalency follows by Theorem 8 .

(2) Similar to the proof of (14), we have

$$
\begin{gathered}
A^{r} \geq B^{r}, \quad A^{p_{0} / 2} B^{r} A^{p_{0} / 2} \leq A^{p_{0} / 2} A^{r} A^{p_{0} / 2}, \\
\left(A^{p_{0} / 2} B^{r} A^{p_{0} / 2}\right)^{\left(p-p_{0}\right) /\left(r+p_{0}\right)} \leq\left(A^{p_{0} / 2} A^{r} A^{p_{0} / 2}\right)^{\left(p-p_{0}\right) /\left(r+p_{0}\right)} .
\end{gathered}
$$

Hence, (16) holds. Since (12) implies (13) without kernel condition, (17) follows by (16).

(3) By (15), there exists the function $d(t)=t+r$ defined on $(0, \infty)$ satisfying $[18$, Lemma $2.6(1)]$. Hence, case $1=p_{0}<p$ of $[18$, Lemma $2.6(2)]$ implies

$$
\begin{aligned}
& \left(A^{r / 2} B A^{r / 2}\right)^{(\min \{p, 2+r\}+r) /(1+r)} \\
& \quad \geq\left(A^{r / 2} B^{p} A^{r / 2}\right)^{(\min \{p, 2+r\}+r) /(p+r)} .
\end{aligned}
$$

So (18) holds by $A \geq B \geq 0$ and (L-H) for $(1+r) /(\min \{p, 2+$ $r\}+r) \in(0,1)$. It is easy to prove (19) in a similar way.

As prompt applications, we show alternate proofs of some Furuta type inequalities.

Theorem 10 (see [19]). Let $\alpha>0, \beta_{0}>0, A \geq 0, B \geq 0$. For $\delta$ such that $-\beta_{0}<\delta \leq \alpha$, if

$$
\left(B^{\beta_{0} / 2} A^{\alpha} B^{\beta_{0} / 2}\right)^{\left(\delta+\beta_{0}\right) /\left(\alpha+\beta_{0}\right)} \geq(\text { resp. } \leq) B^{\delta+\beta_{0}},
$$

then

$$
\left(B^{\beta / 2} A^{\alpha} B^{\beta / 2}\right)^{(\delta+\beta) /(\alpha+\beta)} \geq(\operatorname{resp} . \leq) B^{\delta+\beta},
$$

where $\beta \geq \beta_{0}$. Moreover, for each $\delta^{\prime}>-\alpha$, the function

$$
f(\beta)=\left(A^{\alpha / 2} B^{\beta} A^{\alpha / 2}\right)^{\left(\delta^{\prime}+\alpha\right) /(\beta+\alpha)}
$$

is decreasing (resp., increasing) for $\beta \geq \max \left\{\beta_{0}, \delta^{\prime}\right\}$.

Proof. It is enough to prove the case $\geq$ because the case $\leq$ can be proved in a similar manner. Denote (23) by $A_{1} \geq B_{1}$; that is,

$$
A_{1}=\left(B^{\beta_{0} / 2} A^{\alpha} B^{\beta_{0} / 2}\right)^{\left(\delta+\beta_{0}\right) /\left(\alpha+\beta_{0}\right)}, \quad B_{1}=B^{\delta+\beta_{0}} .
$$

For $1 \geq r_{1}>0, p_{1} \geq 1$, by (19) of Theorem 9, we have

$$
\left(B_{1}^{r_{1} / 2} A_{1}^{p} B_{1}^{r_{1} / 2}\right)^{\left(1+r_{1}\right) /\left(p_{1}+r_{1}\right)} \geq B_{1}^{1+r_{1}} .
$$

By putting $p_{1}=\left(\alpha+\beta_{0}\right) /\left(\delta+\beta_{0}\right)$, the inequality above becomes

$$
\begin{aligned}
& \left(B^{\left(\beta_{0}+\left(\delta+\beta_{0}\right) r_{1}\right) / 2} A^{\alpha} B^{\left(\beta_{0}+\left(\delta+\beta_{0}\right) r_{1}\right) / 2}\right)^{\left(\delta+\beta_{0}+\left(\delta+\beta_{0}\right) r_{1}\right) /\left(\alpha+\beta_{0}+\left(\delta+\beta_{0}\right) r_{1}\right)} \\
& \geq B^{\delta+\beta_{0}+\left(\delta+\beta_{0}\right) r_{1}} .
\end{aligned}
$$

This implies that (24) holds for $\beta_{0} \leq \beta \leq \beta_{1}=\beta_{0}+(\delta+$ $\left.\beta_{0}\right)$. Denote $A_{2}=\left(B^{\beta_{1} / 2} A^{\alpha} B^{\beta_{1} / 2}\right)^{\left(\delta+\beta_{1}\right) /\left(\alpha+\beta_{1}\right)}$ and $B_{2}=B^{\delta+\beta_{1}}$; repeating this process, (24) holds for $\beta \geq \beta_{0}$.

For each $\delta^{\prime}>-\alpha, \beta_{1} \geq \max \left\{\beta_{0}, \delta^{\prime}\right\}$, by (24) and (L-H),

$$
\begin{gathered}
\left(B^{\beta_{1} / 2} A^{\alpha} B^{\beta_{1} / 2}\right)^{\left(\delta+\beta_{1}\right) /\left(\alpha+\beta_{1}\right)} \geq B^{\delta+\beta_{1}}, \\
\left(B^{\beta_{1} / 2} A^{\alpha} B^{\beta_{1} / 2}\right)^{v /\left(\alpha+\beta_{1}\right)} \geq B^{v},
\end{gathered}
$$

where $0<v \leq \delta+\beta_{0}$. This together with Theorem 8 and $(\mathrm{L}-\mathrm{H})$ deduce that

$$
\begin{gathered}
\left(A^{\alpha / 2} B^{\beta_{1}} A^{\alpha / 2}\right)^{\left(v+\beta_{1}+\alpha\right) /\left(\beta_{1}+\alpha\right)} \\
\geq\left(A^{\alpha / 2} B^{v+\beta_{1}} A^{\alpha / 2}\right)^{\left(v+\beta_{1}+\alpha\right) /\left(v+\beta_{1}+\alpha\right)}, \\
\left(A^{\alpha / 2} B^{\beta_{1}} A^{\alpha / 2}\right)^{\left(\delta^{\prime}+\alpha\right) /\left(\beta_{1}+\alpha\right)} \geq\left(A^{\alpha / 2} B^{\beta_{2}} A^{\alpha / 2}\right)^{\left(\delta^{\prime}+\alpha\right) /\left(\beta_{2}+\alpha\right)},
\end{gathered}
$$

where $\beta_{2}=v+\beta_{1} \in\left(\beta_{1}, \delta+\beta_{0}+\beta_{1}\right]$. So, the monotonicity of the function $f(\beta)$ holds.

It should be pointed out that, if $\delta=0$ and $0<\beta<\beta_{0}$, the assertion that (23) ensures (24) is not true [15, Theorem 2.8].

Theorem 11 (see [15]). Given any positive numbers $p, r, p_{1}$, and $r_{1}$ with $r_{1}>r$, there exist invertible positive operators $H$ and $K$ such that

$$
\begin{aligned}
H^{r_{1}} & \geq\left(H^{r_{1} / 2} K^{p_{1}} H^{r_{1} / 2}\right)^{r_{1} /\left(p_{1}+r_{1}\right)}, \\
a H^{r} & \geq\left(H^{r / 2} K^{p} H^{r / 2}\right)^{r /(p+r)},
\end{aligned}
$$

where $a$ is an arbitrary positive number.

Alternate Proof of Theorem 2. The case $r \geq 0$ and $0<p \leq 1$ of Theorem 2 follows by (L-H) directly. Theorem 9(3) means the case $0<r \leq 1$ and $p>1$ of Theorem 2; this together with Theorem 10 implies the case $r>0$ and $p>1$ of Theorem 2 . So, the proof is complete.

The proof above says that the original form of Furuta inequality (Theorem 2) is a composition of (L-H), Theorems 9 and 10 . The proof here is independent of the invertibility of the operators $A$ and $B$.

\section{A Unified Satellite Theorem of Grand Furuta Inequalities}

Denote $:=\left(A^{t / 2} B^{p} A^{t / 2}\right)^{1 /(p+t)}$, where $p+t \neq 0$. 
Theorem 12. Let $-1 \leq t<0, p \geq 1, \widetilde{p} \geq 1, C \geq A \geq B \geq 0$ with $A>0$.

(1) If $r \geq-t, s \geq 1,1 \leq p_{0} \leq p$ and $1 \leq p_{0} \leq \widetilde{p}$, then

$$
\begin{aligned}
& \left(A^{r / 2}\left(A^{t / 2} B^{p} A^{t / 2}\right)^{s} A^{r / 2}\right)^{\left(p_{0}+t+r\right) /((p+t) s+r)} \\
& \quad \leq A^{(r+t) / 2} B^{p_{0}} A^{(r+t) / 2} \leq A^{r / 2}\left(A^{t / 2} B^{\tilde{p}} A^{t / 2}\right)^{\left(p_{0}+t\right) /(\tilde{p}+t)} A^{r / 2} .
\end{aligned}
$$

(2) If $r \geq-t$ and $s \geq 1$, then

$$
\begin{gathered}
\left(C^{r / 2}\left(A^{t / 2} B^{p} A^{t / 2}\right)^{s} C^{r / 2}\right)^{(1+t+r) /((p+t) s+r)} \\
\leq C^{r / 2}\left(A^{t / 2} B^{p} A^{t / 2}\right)^{(1+t) /(p+t)} C^{r / 2} .
\end{gathered}
$$

The case $C=A$ of Theorem 12(2) is just Theorem 7. The special case $p_{0}=1$ of Theorem 12(1) implies the result below.

Corollary 13. Let $-1 \leq t<0, p \geq 1, \widetilde{p} \geq 1, A \geq B \geq 0$ with $A>0$. If $r \geq-t$ and $s \geq 1$, then

$$
\begin{aligned}
& \left(A^{r / 2}\left(A^{t / 2} B^{p} A^{t / 2}\right)^{s} A^{r / 2}\right)^{(1+t+r) /((p+t) s+r)} \\
& \quad \leq A^{(r+t) / 2} B A^{(r+t) / 2} \leq A^{r / 2}\left(A^{t / 2} B^{\tilde{p}} A^{t / 2}\right)^{(1+t) /(\tilde{p}+t)} A^{r / 2} .
\end{aligned}
$$

It is obvious that the special case $p=\widetilde{p}$ of Corollary 13 is a unified result of Theorems 6 and 7; that is, it is an extension of Theorems 6 and 7. So, we call Theorem 12 a unified satellite theorem of grand Furuta inequality (Theorem 5).

In order to give a proof, we prepare some results in advance.

Lemma 14 (see [18]). Let $-1 \leq t<0, p \geq 1$ and $s \geq 1$. Then $C \geq A \geq B \geq 0$ with $A>0$ ensures that the function

$$
f(s)=\left(C^{-t / 2}\left(A^{t / 2} B^{p} A^{t / 2}\right)^{s} C^{-t / 2}\right)^{1 /((p+t) s-t)}
$$

is decreasing for $s \geq 1$. In particular,

$$
C \geq\left(C^{-t / 2}\left(A^{t / 2} B^{p} A^{t / 2}\right)^{s} C^{-t / 2}\right)^{1 /((p+t) s-t)} .
$$

Lemma 15 (see [18]). Let $-1 \leq t<0, p \geq 1, r \geq-t, 1 \leq s_{0}<s$ and $\delta=\min \left\{(p+t) s, 2(p+t) s_{0}\right\}$. Then $C \geq A \geq B \geq 0$ with $A>0$ ensures

$$
\begin{aligned}
& \left(C^{r / 2} D^{(p+t) s_{0}} C^{r / 2}\right)^{(\delta+r) /\left((p+t) s_{0}+r\right)} \\
& \quad \geq\left(C^{r / 2} D^{(p+t) s} C^{r / 2}\right)^{(\delta+r) /((p+t) s+r)} .
\end{aligned}
$$

Lemma 16. Let $r<0, A>0$ and $B \geq 0$. Then the following assertion (1) implies (2).
(1) There exists an increasing function $d(p):(-r, \infty) \rightarrow$ $(0, \infty)$ such that, for each $p_{0}>-r$, if $p_{0}<p \leq p_{0}+$ $d\left(p_{0}\right)$, then

$$
\left(A^{r / 2} B^{p_{0}} A^{r / 2}\right)^{(p+r) /\left(p_{0}+r\right)} \geq(\text { resp. } \leq)\left(A^{r / 2} B^{p} A^{r / 2}\right)^{(p+r) /(p+r)} .
$$

(2) The function $d(p):(-r, \infty) \rightarrow(0, \infty)$ in (1) satisfies that, for each $p_{0}>-r$, if $p_{0}<p$, then

$$
\begin{aligned}
& \left(A^{r / 2} B^{p_{0}} A^{r / 2}\right)^{\left(\min \left\{p, p_{0}+d\left(p_{0}\right)\right\}+r\right) /\left(p_{0}+r\right)} \\
& \quad \geq(\text { resp. } \leq)\left(A^{r / 2} B^{p} A^{r / 2}\right)^{\left(\min \left\{p, p_{0}+d\left(p_{0}\right)\right\}+r\right) /(p+r)} .
\end{aligned}
$$

Lemma 16 is a complement to [18, Lemma 2.6].

Proof. It is sufficient to prove the case $\geq$ for the case $\leq$ can be proved in a similar manner. For each $p_{0}>0$ and $p_{0}<p$, if $p \leq p_{0}+d\left(p_{0}\right)$, then (2) follows by (1) immediately. Suppose that $p_{n}<p \leq p_{n+1}=p_{n}+d\left(p_{n}\right)$ for some positive integer $n$ and $p_{1}=p_{0}+d\left(p_{0}\right)$. By $(1)$, for $k=0,1, \ldots, n-1$, we have

$$
\begin{aligned}
\left(A^{r / 2} B^{p_{k}} A^{r / 2}\right)^{\left(p_{k+1}+r\right) /\left(p_{k}+r\right)} & \geq\left(A^{r / 2} B^{p_{k+1}} A^{r / 2}\right)^{\left(p_{k+1}+r\right) /\left(p_{k+1}+r\right)}, \\
\left(A^{r / 2} B^{p_{n}} A^{r / 2}\right)^{(p+r) /\left(p_{n}+r\right)} & \geq\left(A^{r / 2} B^{p} A^{r / 2}\right)^{(p+r) /(p+r)} .
\end{aligned}
$$

Noting that $\left(\left(p_{1}+r\right) /\left(p_{k+1}+r\right)\right) \in[0,1]$ and $\left(\left(p_{1}+r\right) /(p+r)\right) \in$ $[0,1]$, these together with (L-H) deduce that

$$
\begin{aligned}
\left(A^{r / 2} B^{p_{0}} A^{r / 2}\right)^{\left(p_{1}+r\right) /\left(p_{0}+r\right)} & \geq\left(A^{r / 2} B^{p_{1}} A^{r / 2}\right)^{\left(p_{1}+r\right) /\left(p_{1}+r\right)} \\
& \geq \cdots \geq\left(A^{r / 2} B^{p_{n}} A^{r / 2}\right)^{\left(p_{1}+r\right) /\left(p_{n}+r\right)} \\
& \geq\left(A^{r / 2} B^{p} A^{r / 2}\right)^{\left(p_{1}+r\right) /(p+r)} .
\end{aligned}
$$

Therefore, the function $d$ in (1) satisfies (2).

Lemma 17. Let $0>r \geq-1, p>p_{0}>-r$ and $\beta=$ $\min \left\{p, 2 p_{0}+r\right\}$; then $A \geq B \geq 0$ with $A>0$ ensures

$$
\left(A^{r / 2} B^{p_{0}} A^{r / 2}\right)^{(\beta+r) /\left(p_{0}+r\right)} \leq\left(A^{r / 2} B^{p} A^{r / 2}\right)^{(\beta+r) /(p+r)} .
$$

Proof. Firstly, we prove the case $p \leq 2 p_{0}+r$ of Lemma 17. By [10, Lemma 1], (42) is equivalent to

$$
\begin{gathered}
A^{r / 2} B^{p_{0} / 2}\left(B^{p_{0} / 2} A^{r} B^{p_{0} / 2}\right)^{\left(p-p_{0}\right) /\left(p_{0}+r\right)} B^{p_{0} / 2} A^{r / 2} \\
\leq A^{r / 2} B^{p_{0} / 2} B^{p-p_{0}} B^{p_{0} / 2} A^{r / 2} .
\end{gathered}
$$

On the other hand, $\left(B^{p_{0} / 2} A^{r} B^{p_{0} / 2}\right)^{\left(p-p_{0}\right) /\left(p_{0}+r\right)} \leq B^{p-p_{0}}$ holds by Loewner-Heinz inequality for $\left(\left(p-p_{0}\right) /\left(p_{0}+r\right)\right) \in(0,1]$. So (42) holds for $p \leq 2 p_{0}+r$.

Now, it is proved that (42) holds when $-r<p_{0}<$ $p \leq 2 p_{0}+r$. Meanwhile, it is easy to see that the increasing function $d(t)=t+r$ satisfies (1) of Lemma 16, so (42) holds for $-r<p_{0}<p$. 
Proof of Theorem 12. By the case $s_{0}=1$ of Lemma 15 and (L$\mathrm{H})$ for $\left(\left(p_{0}+t+r\right) /(\delta+r)\right) \in[0,1]$,

$$
\begin{aligned}
& \left(C^{r / 2}\left(A^{t / 2} B^{p} A^{t / 2}\right)^{s} C^{r / 2}\right)^{\left(p_{0}+t+r\right) /((p+t) s+r)} \\
& \quad \leq\left(C^{r / 2}\left(A^{t / 2} B^{p} A^{t / 2}\right) C^{r / 2}\right)^{\left(p_{0}+t+r\right) /((p+t)+r)} .
\end{aligned}
$$

(1) For $1 \leq p_{0} \leq p$, Theorem 3 and (L-H) deduce that

$$
\left(A^{(r+t) / 2} B^{p} A^{(r+t) / 2}\right)^{\left(p_{0}+t+r\right) /(p+r+t)} \leq A^{(r+t) / 2} B^{p_{0}} A^{(r+t) / 2} .
$$

Meanwhile, for $-1 \leq t<0$ and $1 \leq p_{0} \leq \widetilde{p}$, Lemma 17 and (L-H) imply

$$
A^{t / 2} B^{p_{0}} A^{t / 2} \leq\left(A^{t / 2} B^{\widetilde{p}} A^{t / 2}\right)^{\left(p_{0}+t\right) /(\tilde{p}+t)}
$$

Hence, (1) follows by the case $C=A$ of (44), (45), and (46).

(2) By (L-H), (44), $f(1) \leq C$, Theorem 3 and Lemma 17 ensure

$$
\begin{aligned}
& \left(C^{r / 2}\left(A^{t / 2} B^{p} A^{t / 2}\right)^{s} C^{r / 2}\right)^{(1+t+r) /((p+t) s+r)} \\
& \leq\left(C^{r / 2}\left(A^{t / 2} B^{p} A^{t / 2}\right) C^{r / 2}\right)^{(1+t+r) /((p+t)+r)}
\end{aligned}
$$

by (44) and (L-H)

$$
\begin{gathered}
=\left(C^{(r+t) / 2}\left(C^{-t / 2} A^{t / 2} B^{p} A^{t / 2} C^{-t / 2}\right)^{(1 / p) p}\right. \\
\left.\quad \times C^{(r+t) / 2}\right)^{(1+r+t) /(p+r+t)} \\
=\left(C^{(r+t) / 2}(f(1))^{p} C^{(r+t) / 2}\right)^{(1+r+t) /(p+r+t)} \\
\leq\left(C^{(r+t) / 2}(f(1))^{1} C^{(r+t) / 2}\right)^{(1+r+t) /(1+r+t)}
\end{gathered}
$$

by $f(1) \leq C$, Theorem 3 and $(\mathrm{L}-\mathrm{H})$

$$
\begin{aligned}
& =C^{r / 2} C^{t / 2}(f(1))^{1} C^{t / 2} C^{r / 2} \\
& \leq C^{r / 2}\left(C^{t / 2}(f(1))^{p} C^{t / 2}\right)^{(1+t) /(p+t)} C^{r / 2}
\end{aligned}
$$

by $f(1) \leq C$, Lemma 17 and $(\mathrm{L}-\mathrm{H})$

$$
=C^{r / 2}\left(A^{t / 2} B^{p} A^{t / 2}\right)^{(1+t) /(p+t)} C^{r / 2} \text {. }
$$

The $f(s)$ above is the same as the function $f(s)$ in Lemma 14.

\section{Riccati Type Operator Equations}

Yuan and Gao [15] discussed the Riccati type equation:

$$
K^{p}=H^{\delta / 2} T^{1 / 2}\left(T^{1 / 2} H^{\delta+r} T^{1 / 2}\right)^{(p-\delta) /(\delta+r)} T^{1 / 2} H^{\delta / 2} .
$$

Theorem 18 (see [15]). Let $H \geq 0, K \geq 0$ and assume that ker $H=\{0\}$.

(1) The following statements are equivalent for each $p>0$, $r>0$ and $p \geq \delta \geq 0$.

(a) $a H^{\delta+r} \geq\left(H^{r / 2} K^{p} H^{r / 2}\right)^{(\delta+r) /(p+r)}$ for some $a \geq 0$.

(b) There exists a unique operator $T \geq 0$ that satisfies $\|T\| \leq a$ and (48).

If in additional $H$ is invertible, (1) holds for $p \geq \delta>-r$.

(2) If there exists $T \geq 0$ satisfying (48) for fixed $p>0$, $r>0$ and $p \geq \delta \geq 0$, then, for $p_{1} \geq p$ and $r_{1} \geq r$, there exists $T_{1} \geq 0$ satisfying

$$
K^{p_{1}}=H^{\delta / 2} T_{1}^{1 / 2}\left(T_{1}^{1 / 2} H^{\delta+r_{1}} T_{1}^{1 / 2}\right)^{\left(p_{1}-\delta\right) /\left(\delta+r_{1}\right)} T_{1}^{1 / 2} H^{\delta / 2} .
$$

One of the applications of Riccati equation (48) is to show that the inclusion relations among class $A(p, r)$ operators are strict [15, Theorem 3.1]. Recently, there are some developments on operator equations including the following equation (see $[16,20])$ :

$$
K^{p}=H^{\delta / 2} T^{1 / 2}\left(T^{1 / 2} H^{\delta+r} T^{1 / 2}\right)^{(1 / w)-1} T^{1 / 2} H^{\delta / 2} .
$$

Obviously, the special case $w=(\delta+r) /(p+r)$ of $(50)$ is just (48).

Theorem 19 (see [16]). Let $H \geq 0, K \geq 0$ and assume that ker $H=\{0\}$. The following statements are equivalent for each $p>0, r>0, \delta \geq 0$ and $0<w \leq 1$.

(1) $a H^{\delta+r} \geq\left(H^{r / 2} K^{p} H^{r / 2}\right)^{w}$ for some $a \geq 0$.

(2) There exists a unique operator $T \geq 0$ which satisfies $\|T\| \leq a$ and (50).

If in additional $H$ is invertible, the condition $\delta \geq 0$ can be replaced with $\delta \in \mathscr{R}$ where $\mathscr{R}$ means the set of all real numbers, and if $H$ and $K$ are both invertible, the conditions $\delta \geq 0$ and $0<w \leq 1$ can be replaced with $\delta \in \mathscr{R}$ and $w \neq 0$.

The case $w=(\delta+r) /(p+r)$ of Theorem 19 is a generalization of Theorem 18(1). In this section, we give a generalization of Theorem 18(2).

Lemma 20. Let $\alpha>0, \beta_{0}>0, \alpha^{\prime}>0, \beta_{0}^{\prime}>0, A \geq 0, B \geq 0$. For $\delta$ and $\delta^{\prime}$ such that $\delta \in\left(-\beta_{0}, \infty\right)$ and $\delta^{\prime} \in\left(-\beta_{0}^{\prime}, \alpha^{\prime}\right]$, if

$$
\left(B^{\beta_{0} / 2} A^{\alpha} B^{\beta_{0} / 2}\right)^{\left(\delta^{\prime}+\beta_{0}^{\prime}\right) /\left(\alpha^{\prime}+\beta_{0}^{\prime}\right)} \geq(\text { resp. } \leq) B^{\delta+\beta_{0}},
$$

then, for $\beta \geq \beta_{0}$,

$$
\left(B^{\beta / 2} A^{\alpha} B^{\beta / 2}\right)^{\left(\delta^{\prime}+\beta^{\prime}\right) /\left(\alpha^{\prime}+\beta^{\prime}\right)} \geq(\text { resp. } \leq) B^{\delta+\beta},
$$

where $\beta^{\prime}=\left(\left(\beta\left(\delta^{\prime}+\beta_{0}^{\prime}\right)+\beta_{0}^{\prime} \delta-\beta_{0} \delta^{\prime}\right) /\left(\delta+\beta_{0}\right)\right)\left(\geq \beta_{0}^{\prime}\right)$.

The case $\alpha=\alpha^{\prime}, \beta_{0}=\beta_{0}^{\prime}$ and $\delta=\delta^{\prime}$ of Lemma 20 implies Theorem 10. The case $\alpha=\alpha^{\prime}, \beta_{0}=\beta_{0}^{\prime}$ and $\delta=\delta^{\prime}=0$ of Lemma 20 implies Yanagida's result [21, Proposition 4]. 
Proof. It is enough to prove the case $\geq$ because the case $\leq$ can be proved in a similar manner. Denote (51) by $A_{1} \geq B_{1}$; that is,

$$
A_{1}=\left(B^{\beta_{0} / 2} A^{\alpha} B^{\beta_{0} / 2}\right)^{\left(\delta^{\prime}+\beta_{0}^{\prime}\right) /\left(\alpha^{\prime}+\beta_{0}^{\prime}\right)}, \quad B_{1}=B^{\delta+\beta_{0}} .
$$

For $r_{1}>0, p_{1} \geq 1$, by (FI) (Theorem 2), we have

$$
\left(B_{1}^{r_{1} / 2} A_{1}^{p_{1}} B_{1}^{r_{1} / 2}\right)^{\left(1+r_{1}\right) /\left(p_{1}+r_{1}\right)} \geq B_{1}^{1+r_{1}} .
$$

By putting $p_{1}=\left(\left(\alpha^{\prime}+\beta_{0}^{\prime}\right) /\left(\delta^{\prime}+\beta_{0}^{\prime}\right)\right)(\geq 1)$, the inequality above becomes

$$
\begin{aligned}
& \left(B^{\left(\beta_{0}+\left(\delta+\beta_{0}\right) r_{1}\right) / 2} A^{\alpha}\right. \\
& \left.\quad \times B^{\left(\beta_{0}+\left(\delta+\beta_{0}\right) r_{1}\right) / 2}\right)^{\left(\delta^{\prime}+\beta_{0}^{\prime}+\left(\delta^{\prime}+\beta_{0}^{\prime}\right) r_{1}\right) /\left(\alpha^{\prime}+\beta_{0}^{\prime}+\left(\delta^{\prime}+\beta_{0}^{\prime}\right) r_{1}\right)} \\
& \quad \geq B^{\delta+\beta_{0}+\left(\delta+\beta_{0}\right) r_{1}} .
\end{aligned}
$$

Denote $\beta=\beta_{0}+\left(\delta+\beta_{0}\right) r_{1}$ and $\beta^{\prime}=\beta_{0}^{\prime}+\left(\delta^{\prime}+\beta_{0}^{\prime}\right) r_{1}$; then $\beta \geq \beta_{0}$ and $\beta^{\prime}=\left(\left(\beta\left(\delta^{\prime}+\beta_{0}^{\prime}\right)+\beta_{0}^{\prime} \delta-\beta_{0} \delta^{\prime}\right) /\left(\delta+\beta_{0}\right)\right) \geq \beta_{0}^{\prime}$, so that (52) holds by the inequality above.

Theorem 21. Let $H \geq 0, K \geq 0$ and assume that ker $H=\{0\}$. For each $p>0, r>0$ and $\delta \geq 0$, if there exists $T \geq 0$ satisfying the equation

$$
K^{p}=H^{\delta / 2} T^{1 / 2}\left(T^{1 / 2} H^{\delta+r} T^{1 / 2}\right)^{\left(p^{\prime}-\delta^{\prime}\right) /\left(\delta^{\prime}+r^{\prime}\right)} T^{1 / 2} H^{\delta / 2},
$$

where $p^{\prime}>0, r^{\prime}>0$ and $-r^{\prime}<\delta^{\prime} \leq p^{\prime}$. Then, for $r_{1} \geq r$ and $r_{1}^{\prime}=\left(\left(r_{1}\left(\delta^{\prime}+r^{\prime}\right)+r^{\prime} \delta-r \delta^{\prime}\right) /(\delta+r)\right)\left(\geq r^{\prime}\right)$, there exists $T_{1} \geq 0$ satisfying

$$
K^{p}=H^{\delta / 2} T_{1}^{1 / 2}\left(T_{1}^{1 / 2} H^{\delta+r_{1}} T_{1}^{1 / 2}\right)^{\left(p^{\prime}-\delta^{\prime}\right) /\left(\delta^{\prime}+r_{1}^{\prime}\right)} T_{1}^{1 / 2} H^{\delta / 2} .
$$

If $H$ is invertible, the condition $\delta \geq 0$ can be replaced with $\delta>-r$.

Proof. By the assumption, (1) of Theorem 19 holds for some $a>0$; that is,

$$
\begin{aligned}
\left(a^{1 /(\delta+r(1-w))} H\right)^{\delta+r} & \\
\geq & \left(\left(a^{1 /(\delta+r(1-w))} H\right)^{r / 2}\right. \\
& \left.\quad \times K^{p}\left(a^{1 /(\delta+r(1-w))} H\right)^{r / 2}\right)^{w} .
\end{aligned}
$$

So, the following holds by Lemma 20:

$$
\begin{aligned}
& \left(a^{1 /(\delta+r(1-w))} H\right)^{\delta+r_{1}} \\
& \geq\left(\left(a^{1 /(\delta+r(1-w))} H\right)^{r_{1} / 2}\right. \\
& \left.\quad \times K^{p}\left(a^{1 /(\delta+r(1-w))} H\right)^{r_{1} / 2}\right)^{\left(\delta^{\prime}+r_{1}^{\prime}\right) /\left(p^{\prime}+r_{1}^{\prime}\right)},
\end{aligned}
$$

where $r_{1}^{\prime}=\left(\left(r_{1}\left(\delta^{\prime}+r^{\prime}\right)+r^{\prime} \delta-r \delta^{\prime}\right) /(\delta+r)\right)\left(\geq r^{\prime}\right)$; that is,

$$
a^{\left(\delta+r_{1}\left(1-w_{1}\right)\right) /(\delta+r(1-w))} H^{\delta+r_{1}} \geq\left(H^{r_{1} / 2} K^{p} H^{r_{1} / 2}\right)^{w_{1}},
$$

where $w_{1}=\left(\left(\delta^{\prime}+r_{1}^{\prime}\right) /\left(p^{\prime}+r_{1}^{\prime}\right)\right) \in(0,1]$. Hence, $(57)$ is solvable by Theorem 19 .

The result below is the case $r=r^{\prime}$ and $\delta=\delta^{\prime}$ of Theorem 21.

Corollary 22. Let $H \geq 0, K \geq 0$ and assume that ker $H=\{0\}$. For each $p>0, r>0$ and $\delta \geq 0$, if there exists $T \geq 0$ satisfying the equation

$$
K^{p}=H^{\delta / 2} T^{1 / 2}\left(T^{1 / 2} H^{\delta+r} T^{1 / 2}\right)^{\left(p^{\prime}-\delta\right) /(\delta+r)} T^{1 / 2} H^{\delta / 2},
$$

where $p^{\prime}>0$ and $p^{\prime} \geq \delta$. Then, for $r_{1} \geq r$, there exists $T_{1} \geq 0$ satisfying

$$
K^{p}=H^{\delta / 2} T_{1}^{1 / 2}\left(T_{1}^{1 / 2} H^{\delta+r_{1}} T_{1}^{1 / 2}\right)^{\left(p^{\prime}-\delta\right) /\left(\delta+r_{1}\right)} T_{1}^{1 / 2} H^{\delta / 2} .
$$

If $H$ is invertible, the condition $\delta \geq 0$ can be replaced with $\delta>-r$.

It is obvious that Corollary 22 is a generalization of the case $p_{1}=p$ of Theorem 18(2).

\section{Conflict of Interests}

The authors declare that there is no conflict of interests regarding the publication of this paper.

\section{Acknowledgments}

This work was supported in part by the National Natural Science Foundation of China (11301155) and Project of Education Department of Henan Province of China (2012GGJS-061), and Project of Science and Technology Department of Henan Province of China (142300410143).

\section{References}

[1] R. V. Kadison and J. R. Ringrose, Fundamentals of the Theory of Operator Algebras, American Mathematical Society, Providence, RI, USA, 1997.

[2] T. Furuta, Invitation to Linear Operators, Taylor \& Francis, London, UK, 2001.

[3] T. Furuta, " $A \geq B \geq 0$ assures $\left(B^{r} A^{p} B^{r}\right)^{1 / q} \geq B^{p+2 r / q}$ for $r \geq$ $0, p \geq 0, q \geq 1$ with $(1+2 r) q \geq p+2 r$," Proceedings of the American Mathematical Society, vol. 101, no. 1, pp. 85-88, 1987.

[4] J. Yuan and Z. Gao, "Complete form of Furuta inequality," Proceedings of the American Mathematical Society, vol. 136, no. 8, pp. 2859-2867, 2008.

[5] J.-C. Bourin and E. Ricard, "An asymmetric Kadison's inequality," Linear Algebra and Its Applications, vol. 433, no. 3, pp. 499510, 2010.

[6] S. R. Garcia, "Aluthge transforms of complex symmetric operators," Integral Equations and Operator Theory, vol. 60, no. 3, pp. 357-367, 2008. 
[7] V. Lauric, " $\left(C_{p}, \alpha\right)$-hyponormal operators and trace-class selfcommutators with trace zero," Proceedings of the American Mathematical Society, vol. 137, no. 3, pp. 945-953, 2009.

[8] M. Ito, T. Yamazaki, and M. Yanagida, "Generalizations of results on relations between Furuta-type inequalities," Acta Scientiarum Mathematicarum, vol. 69, no. 3-4, pp. 853-862, 2003.

[9] M. Ito and T. Yamazaki, "Relations between two inequalities $\left(B^{r / 2} A^{p} B^{r / 2}\right)^{r / p+r} \geq B^{r}$ and $\left(A^{p / 2} B^{r} A^{p / 2}\right)^{p / p+r} \leq A^{p}$ and their applications," Integral Equations and Operator Theory, vol. 44, no. 4, pp. 442-450, 2002.

[10] T. Furuta, "Extension of the Furuta inequality and Ando-Hiai log-majorization," Linear Algebra and Its Applications, vol. 219, pp. 139-155, 1995.

[11] M. Fujii, R. Nakamoto, and K. Yonezawa, "A satellite of the grand Furuta inequality and Its application," Linear Algebra and Its Applications, vol. 438, no. 4, pp. 1580-1586, 2013.

[12] M. Fujii, E. Kamei, and R. Nakamoto, "Grand Furuta inequality and its variant," Journal of Mathematical Inequalities, vol. 1, no. 3, pp. 437-441, 2007.

[13] P. Lancaster and L. Rodman, The Algebraic Riccati Equation, Academic Press, Oxford, UK, 1995.

[14] G. K. Pedersen and M. Takesaki, "The operator equation THT = K," Proceedings of the American Mathematical Society, vol. 36, pp. 311-312, 1972.

[15] J. Yuan and Z. Gao, "The operator equation $K^{p}=$ $H^{\delta / 2} T^{1 / 2}\left(T^{1 / 2} H^{\delta+r} T^{1 / 2}\right)^{p-\delta / \delta+r} T^{1 / 2} H^{\delta / 2}$ and its applications," Journal of Mathematical Analysis and Applications, vol. 341, no. 2, pp. 870-875, 2008.

[16] J. T. Yuan and C. H. Wang, "Riccati type operator equation and Furuta's question," Mathematical Inequalities \& Application. In press.

[17] J. Yuan, "Furuta inequality and q-hyponormal operators," Operators and Matrices, vol. 4, no. 3, pp. 405-415, 2010.

[18] J. Yuan and G. Ji, "Monotonicity of generalized Furuta type functions," Operators and Matrices, vol. 6, no. 4, pp. 809-818, 2012.

[19] C. S. Yang and J. T. Yuan, "Class $w F(p, r, q)$ operators," Acta Mathematica Scientia A, vol. 27, no. 5, pp. 769-780, 2007.

[20] R. Bhatia and M. Uchiyama, "The operator equation $\sum_{i=0}^{n} A^{n-i} X B^{i}=Y$, Expositiones Mathematicae, vol. 27, no. 3, pp. 251-255, 2009.

[21] M. Yanagida, "Powers of class $w A(s, t)$ operators associated with generalized Aluthge transformation," Journal of Inequalities and Applications, vol. 7, no. 2, pp. 143-168, 2002. 


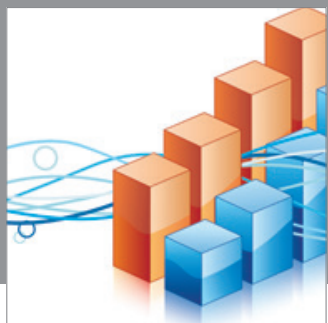

Advances in

Operations Research

mansans

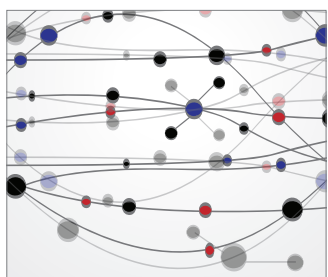

The Scientific World Journal
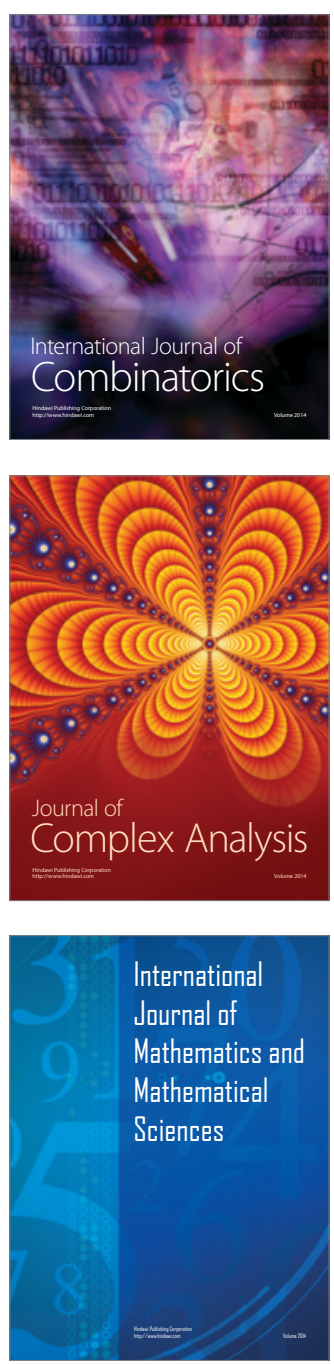
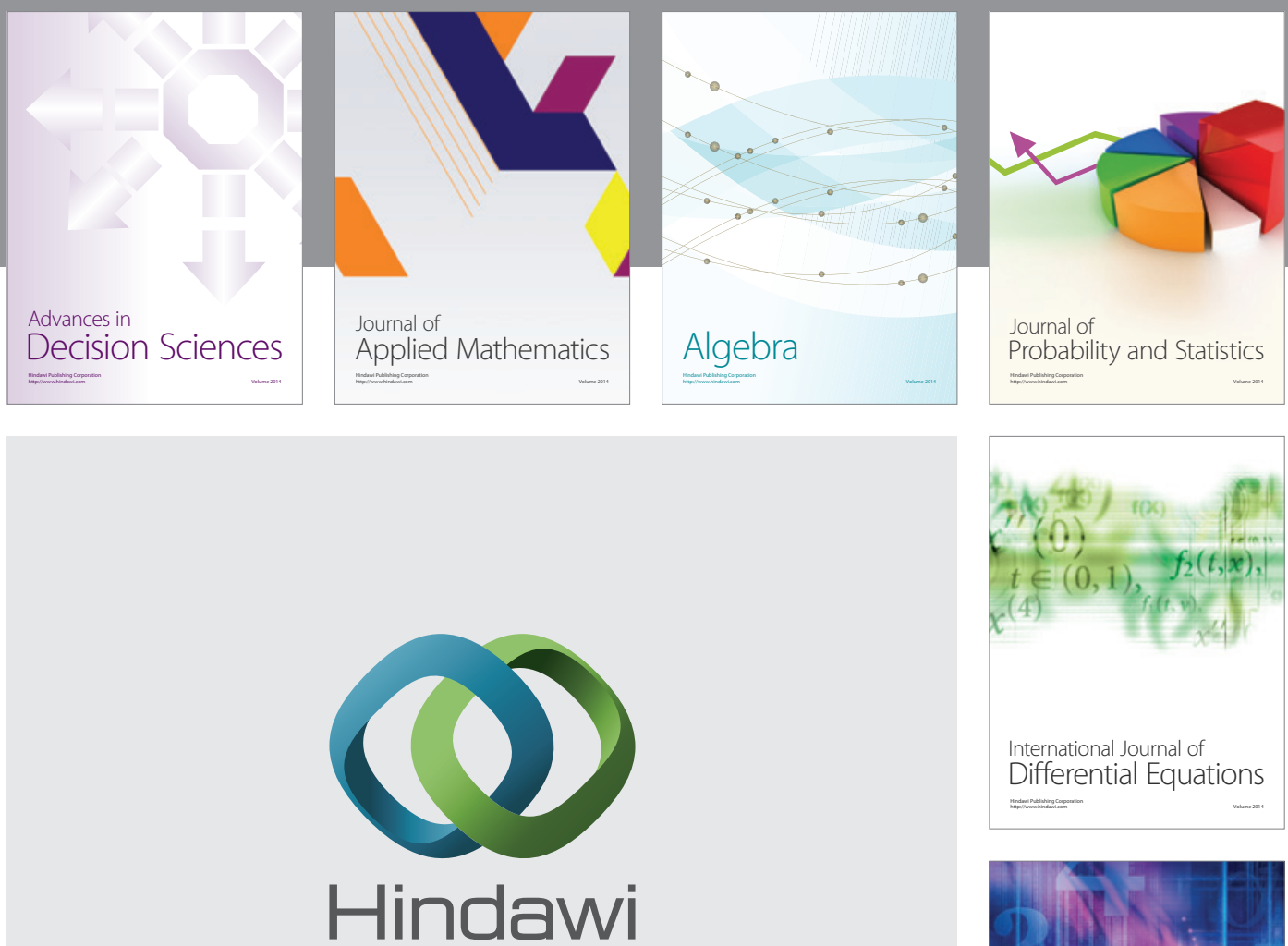

Submit your manuscripts at http://www.hindawi.com
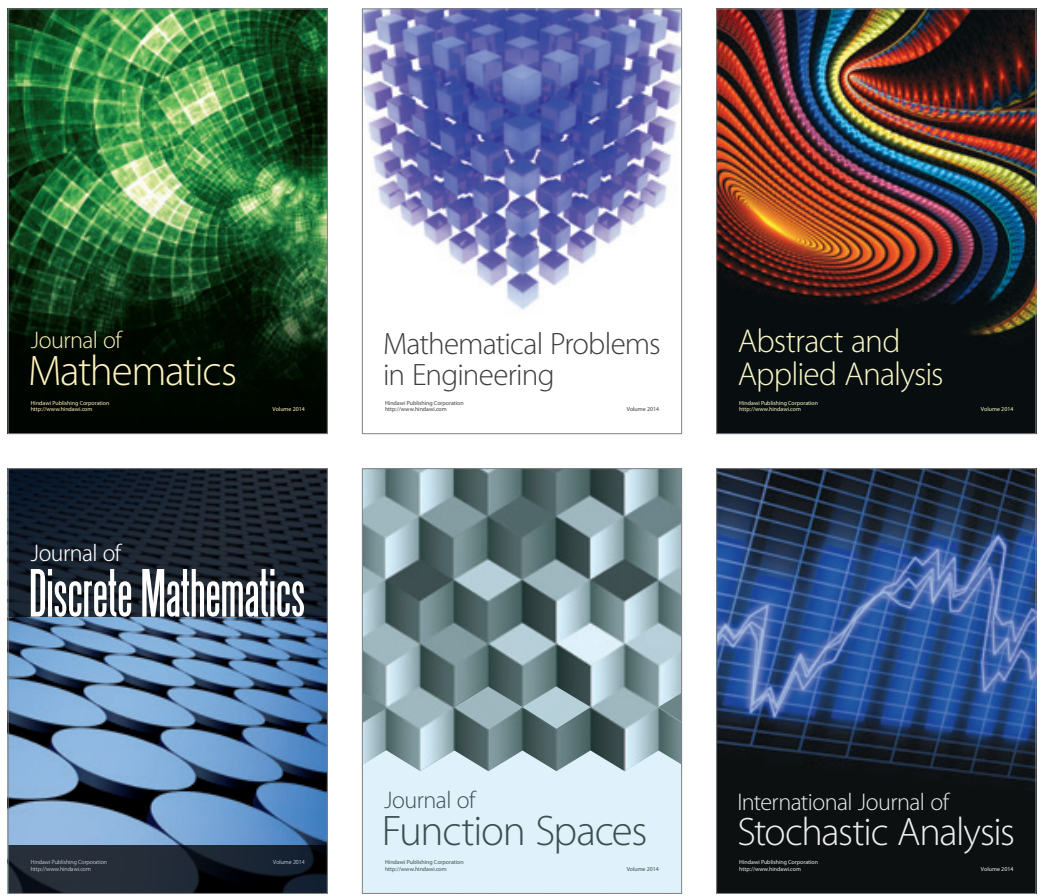

Journal of

Function Spaces

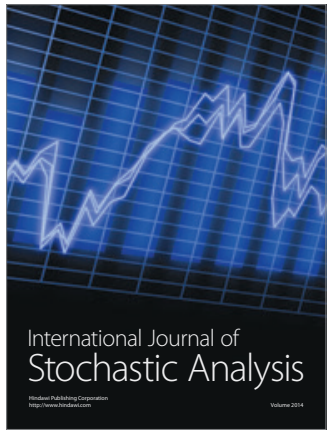

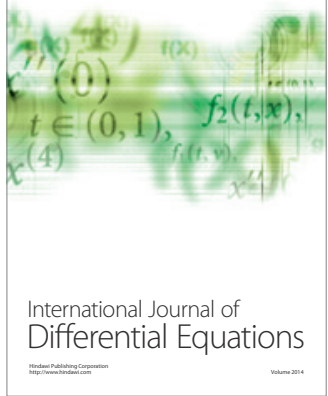
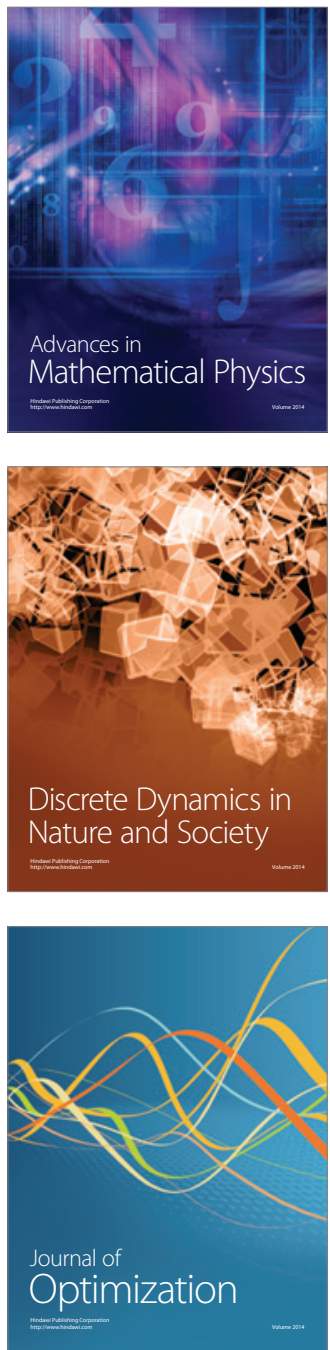